\title{
VIP Deficient Mice Exhibit Resistance to Lipopolysaccharide Induced Endotoxemia with an Intrinsic Defect in Proinflammatory Cellular Responses
}

\author{
Catalina Abad', Yossan-Var Tan ${ }^{1}$, Gardenia Cheung-Lau ${ }^{1,2,3}$, Hiroko Nobuta ${ }^{1}$, James A. Waschek ${ }^{1 *}$ \\ 1 Department of Psychiatry, David Geffen School of Medicine, University of California Los Angeles, Los Angeles, California, United States of America, 2 Department of \\ Pathology and Lab Medicine, David Geffen School of Medicine, University of California Los Angeles, Los Angeles, California, United States of America, 3 Department of \\ Biological Chemistry, David Geffen School of Medicine, University of California Los Angeles, Los Angeles, California, United States of America
}

\begin{abstract}
Vasoactive intestinal peptide (VIP) is a pleiotropic neuropeptide with immunomodulatory properties. The administration of this peptide has been shown to have beneficial effects in murine models of inflammatory diseases including septic shock, rheumatoid arthritis, multiple sclerosis (MS) and Crohn's disease. However, the role of the endogenous peptide in inflammatory disease remains obscure because VIP-deficient mice were recently found to exhibit profound resistance in a model of MS. In the present study, we analyzed the response of female VIP deficient (KO) mice to intraperitoneal lipopolysaccharide (LPS) administration. We observed significant resistance to LPS in VIP KO mice, as evidenced by lower mortality and reduced tissue damage. The increased survival was associated with decreased levels of proinflammatory cytokines (TNF $\alpha$, IL- 6 and IL-12) in sera and peritoneal suspensions of these mice. Moreover, the expression of TNF $\alpha$ and IL- 6 mRNA was reduced in peritoneal cells, spleens and lungs from LPS-treated VIP KO vs. WT mice, suggesting that the resistance might be mediated by an intrinsic defect in the responsiveness of immune cells to endotoxin. In agreement with this hypothesis, peritoneal cells isolated from VIP KO naive mice produced lower levels of proinflammatory cytokines in response to LPS in vitro. Finally, decreased NF- $\mathrm{B}$ pathway activity in peritoneal cells was observed both in vivo and in vitro, as determined by assay of phosphorylated I- $\mathrm{KB}$. The results demonstrate that female VIP KO mice exhibit resistance to LPSinduced shock, explainable in part by the presence of an intrinsic defect in the responsiveness of inflammatory cells to endotoxin.
\end{abstract}

Citation: Abad C, Tan Y-V, Cheung-Lau G, Nobuta H, Waschek JA (2012) VIP Deficient Mice Exhibit Resistance to Lipopolysaccharide Induced Endotoxemia with an Intrinsic Defect in Proinflammatory Cellular Responses. PLoS ONE 7(5): e36922. doi:10.1371/journal.pone.0036922

Editor: Stefan Bereswill, Charité-University Medicine Berlin, Germany

Received April 12, 2012; Accepted April 17, 2012; Published May 17, 2012

Copyright: (c) 2012 Abad et al. This is an open-access article distributed under the terms of the Creative Commons Attribution License, which permits unrestricted use, distribution, and reproduction in any medium, provided the original author and source are credited.

Funding: This work was funded by the National Multiple Sclerosis Society Grants PP1233 and RG3928. The funders had no role in study design, data collection and analysis, decision to publish, or preparation of the manuscript.

Competing Interests: The authors have declared that no competing interests exist.

*E-mail: JWaschek@mednet.ucla.edu

\section{Introduction}

Inflammation is an essential mechanism of self-protection against pathogen invasion and injury, and involves innate and adaptive immune mechanisms [1]. Macrophages, critical effector cells of the innate immune response, play a major role in this process by releasing pro-inflammatory cytokines such as tumor necrosis factor (TNF)- $\alpha$, interleukin (IL)-1 $\beta$, IL-6 and IL-12, and chemokines such as monocyte chemotactic protein (MCP)-1 or macrophage inflammatory protein (MIP)- $1 \alpha$ that promote leukocyte recruitment to the damaged tissues [2]. In turn, the main cellular effectors of innate immunity, macrophages and neutrophils, engulf and digest bacteria, remove cell debris, and produce growth factors to facilitate the healing process $[2,3,4]$. However, the ability of the immune system to mediate direct or indirect killing of cells and pathogens make it a potential threat to host survival. As a consequence, autoimmunity and acute and chronic inflammatory diseases are triggered by immune dysfunction. In this regard, septic shock is a serious acute inflammatory condition often caused by an overwhelming infection that usually leads to impaired perfusion and multiple organ failure [5]. The most frequent microbial agents responsible for septicemia are Gram- negative bacteria. In addition to the release of bacterial toxins, lipopolysaccharide (LPS), a major component of Gram negative bacteria outer membranes, binds preferentially to toll-like receptor (TLR)-4 expressed by many immune and non-immune cell types [6]. This triggers the activation of the nuclear factor kappa B (NF$\kappa \mathrm{B})$ pathway, which causes the release of inflammatory mediators $[7,8]$. Pathology arises when the presence of excessive levels of endotoxin due to bacterial overgrowth leads to a hyperactivation of macrophages and granulocytes that massively invade all tissues and release proinflammatory mediators. This phenomenon can be experimentally mimicked in animals by administration of an exogenous high dose of LPS

Although proper control of the immune response is not always achievable, the organism has developed different endogenous mechanisms to control the inflammatory response and preserve homeostasis [9]. These include the production of soluble mediators such as the anti-inflammatory cytokine IL-10, heat shock proteins, certain prostaglandins, and hormones such as cortisol. Lately, several neuropeptides have been suggested to be immune modulators that dampen the inflammatory response, including the structurally-related peptides VIP and PACAP (vasoactive 
intestinal peptide and pituitary adenylate cyclase-activating polypeptide, respectively) [10]. These natural inhibitors of immunity are currently being tested as potential therapeutic candidates for the treatment of inflammatory diseases.

VIP is a versatile 28-amino acid neuropeptide with thoroughly described anti-inflammatory functions [11,12]. VIP binds mainly to two receptors, named VPAC1 and VPAC2, which belong to the G-coupled protein receptor family and are expressed on many different immune cell types [13]. Numerous in vitro and in vivo studies have demonstrated that VIP, via actions on these receptors, is able to suppress the production and/or release of key molecules for the inflammatory response, such as proinflammatory cytokines, and chemokines [14,15]. In fact, the potential use of VIP as a treatment for acute and chronic inflammatory diseases including septic shock, rheumatoid arthritis, Crohn's disease and multiple sclerosis has been suggested based on positive clinical and pathological outcomes in corresponding murine models of inflammatory diseases $[16,17,18,19]$. In addition, it has been demonstrated that the systemic endogenous levels of this neuropeptide increase in response to an inflammatory challenge like endotoxic shock in humans or LPS-induced endotoxemia in mice [20,21]. As VIP has been reported to be produced by neurons, endocrine and immune cells, multiple tissular and cellular sources can contribute to the increase of VIP after inflammation. For example, it has been recently shown that LPS induces the production of VIP by chromaffin cells from the adrenal medulla [22]. In addition, VIP levels are upregulated in cell suspensions from primary and secondary lymphoid organs in response to LPS treatment [23]. Whether or not this upregulation has an impact on the course of the inflammatory response is largely unknown. VIP-deficient mice $(\mathrm{KO})$ display certain physiological abnormalities such as disrupted circadian rhythms, airway hyperresponsiveness to the cholinergic agonist methacholine, and pulmonary hypertension $[24,25,26]$. In addition, we have recently reported that despite the well-described anti-inflammatory actions of VIP, VIP KO female mice were unexpectedly resistant to experimental autoimmune encephalomyelitis (EAE) induction, with reduced immune cell infiltration of the spinal cord and brain parenchyma [27]. Potential mechanisms for this resistance may include defects in the innate arm of immunity. As a model to test this hypothesis, we investigated the response of VIP KO mice to LPS-induced endotoxemia. We found that female VIP KO mice exhibited reduced mortality in response to LPS. This phenotype was associated with reduced inflammatory damage in the lungs of the $\mathrm{KO}$ mice, and reduced levels of proinflammatory mediators in the sera and peritoneal suspensions. Moreover, we found reduced mRNA expression of TNF $\alpha$ and IL6 in peritoneal cells, spleens and lungs of LPS-treated VIP KO mice. Interestingly, peritoneal cells isolated from naive VIP KO mice produced lower levels of proinflammatory cytokines than wild type [2] cells in response to LPS. Finally, NF- $\kappa$ B activation by LPS was reduced in cells from VIP KO mice.

\section{Materials and Methods}

Mice

All animal studies were approved by the institutional animal research committee of the University of California at Los Angeles (UCLA) Female 6- to 8-week-old mice WT and VIP KO mice on a C57BL/6 background (backcrossed for at least twelve generations) [25] were maintained under specific pathogen-free conditions. Experimental procedures followed the recommendations for animal use and welfare, as dictated by the UCLA Division of
Laboratory Animals and the guidelines from the National Institutes of Health.

\section{Induction of endotoxemia and histology}

Endotoxemia was induced in mice by intraperitoneal (i.p.) injection of $40 \mathrm{mg} / \mathrm{Kg}$ of LPS (Salmonella enteritidis; Sigma, St. Louis, MO), and survival was monitored thereafter for at least one week. For histopathological studies, separate cohorts of mice were sacrificed 24 hours later and lungs were removed and fixed in Bouin's solution for 2 hours. Fixed tissues were then dehydrated and embedded in paraffin, sectioned, and stained with hematoxylin and eosin for morphological examination. The degree of inflammation was scored from 0 to 3 by two independent researchers in a blinded fashion as described [28]: 0 - all alveolar septae thin and delicate, no hemorrhage or fibrosis present; 1 congested alveolar septae in less than $1 / 3$ of the field, mild hemorrhage and fibrosis in less than $1 / 3$ of the field; 2- congested alveolar septae $1 / 3$ to $2 / 3$ of the field, moderate hemorrhage and fibrosis in $1 / 3$ to $2 / 3$ of the field, 3 : congested alveolar septae in more than $2 / 3$ of the field, severe hemorrhage and fibrosis in more than $2 / 3$ of the field.

\section{Collection of sera and peritoneal supernatants}

For cytokine measurements, mice were sacrificed 3 and 6 hours after LPS injection (or 3, 6 and 24 hours for IL-10), blood was collected by intracardiac puncture under anesthesia, and serum was obtained after clotting for 2 hours at room temperature. In order to collect peritoneal suspensions at these time points, mice were injected i.p. with $2 \mathrm{ml}$ of RPMI 1640 (Gibco, Gaithersburg, MD) and fluid was collected after abdominal massage. In both cases, samples were centrifuged for $10 \mathrm{~min}$ at $2000 \mathrm{rpm}$ and supernatants were stored at $-20^{\circ} \mathrm{C}$ until assay.

\section{RNA isolation and Real time-PCR analysis}

RNA was extracted from cells from peritoneal suspension collected as above, and from spleens and lungs at the indicated time points with Trizol (Invitrogen, Carlsbad, CA) as recommended by the manufacturer. RNA was resuspended in diethylpyrocarbonate (DEPG) water and quantified at 260/ $280 \mathrm{~nm}$. cDNA from $1 \mu \mathrm{g}$ of RNA was synthesized with the iScriptTM cDNA synthesis Kit from Bio-Rad (Hercules, CA). Quantitative real-time PCR analysis was performed using the $\mathrm{iQ}^{\mathrm{TM}}$ SYBR Green Supermix from Bio-Rad in triplicate in $25 \mu \mathrm{l}$ reaction volumes. The sequences of the primers used were: mouse HPRT 5'-TGGTGAAAAGGACCTCTCGAA-OH sense and 5'TCAAGGGCATATCGAACAACA-OH antisense, mouse TNF $\alpha$ sense 5'-CGATCACCGCGAAGTTCAGTA-OH and 5'GGTGCGTATGTCTCAGCGTCTT-OH antisense, mouse IL6 5'-TTCGATCGAGTTGCCTTCTTG-OH sense and 5'TTGGGAGTGGTATCCTCTGTGA-OH antisense. The GenBank accession numbers for the PCR products are: HPRT, NM013556; TNF $\alpha$, NM013693; IL-6, NM031168. The amplification conditions were $4 \mathrm{~min}$ at $95^{\circ} \mathrm{C}$ followed by 40 cycles of denaturation at $96^{\circ} \mathrm{C}$ for $20 \mathrm{sec}$, annealing at $\left(60^{\circ} \mathrm{C}\right.$ for IL-6, $62^{\circ} \mathrm{C}$ for $\mathrm{TNF} \alpha$ ), for $30 \mathrm{sec}$ and extension at $72^{\circ} \mathrm{C}$ for $30 \mathrm{sec}$. The specificity of gene amplification was confirmed by sequencing of the PCR products and melting curves analysis. Comparison of specific ratios (gene of interest/house keeping gene) was used to assess differences between groups.

\section{In vitro studies}

For cell culture studies, peritoneal lavage was obtained as described above. Whole peritoneal cells were washed and cultured 
in 96 well tissue culture plates at $2 \times 10^{5}$ cells/well in $200 \mu \mathrm{l} /$ well with RPMI 1640 complete medium (2\% FCS, 2 mM L-glutamine, $100 \mathrm{U} / \mathrm{ml}$ penicillin, and $100 \mu \mathrm{g} / \mathrm{ml}$ streptomycin) and $10 \mathrm{ng} / \mathrm{ml}$ of LPS (Salmonella enteritidis; Sigma) at $37^{\circ} \mathrm{C}$ in a $5 \% \quad \mathrm{CO}_{2}$ humidified atmosphere. Supernatants were collected at different time points, centrifuged at $2000 \mathrm{rpm}$ and stored at $-20^{\circ} \mathrm{C}$ until assay of cytokines by ELISA.

\section{ELISA}

TNF $\alpha$, IL-6, IL-10 and IL-12p40 quantities in serum, peritoneal suspension or culture supernatants were determined by standard sandwich ELISA. Development kits were used according to manufacturer instructions (Peprotech, Rocky Hill, NJ). Briefly, 96 well plates (Corning, NY) were coated with capture antibody $\mathrm{O} / \mathrm{N}$ at room temperature. After blocking for 1 hour with $\mathrm{PBS} / 1 \% \mathrm{BSA}$, samples and standards were added $\mathrm{O} / \mathrm{N}$ at $4^{\circ} \mathrm{C}$. Plates were then incubated with detection antibody for 2 hours, and avidin/peroxidase was added for $30 \mathrm{~min}$. Washing four times with PBS/0.05\% Tween was performed before the addition of all reagents. Finally, plates were incubated with substrate solution and $\mathrm{A}_{405} \mathrm{~nm}$ was measured 30 minutes later with a Bio-Rad microplate reader.

\section{Western blot}

For analysis of freshly isolated peritoneal cells (in vivo), mice were injected with LPS and cells were collected one hour later from peritoneal lavage. For in vitro studies, peritoneal cells were incubated with $10 \mathrm{ng} / \mathrm{ml}$ of LPS as above and collected $15 \mathrm{~min}$ later. In both cases cells were centrifuged and pellets were frozen in dry ice. Pelletted cells were homogenized in RIPA buffer containing protease inhibitor cocktail (Roche, Indianapolis, IN) and phosphatase inhibitor cocktail (Sigma). Concentration of total protein was determined using a BCA kit (GE healthcare, Piscataway, NJ) and $20 \mu \mathrm{g}$ of protein was resolved on a $4-20 \%$ gradient polyacrylamide gel (Invitrogen) and transferred to PVDF membranes (Bio-Rad). Membranes were then incubated with primary antibody for phosphorylated IкB (Santa Cruz, CA), appropriate secondary antibody conjugated to horse-radish peroxidase (Cell Signaling, Danvers, MA), and visualized using ECL plus (GE healthcare). During the steps of blocking, primary and secondary antibody incubations for $\mathrm{p}-\mathrm{I} \kappa \mathrm{B}$, the same phosphatase inhibitor cocktail was added. Films were scanned, and band density was quantified using digital image densitometry analysis (ImageJ; National Institutes of Health, Bethesda, MD).

\section{Statistical analysis}

Graphs were created with the GraphPad 4.0 Prism software. All results were expressed as means \pm SEM. Survival curves were analyzed by Logrank (Mantel-Haenszel) test and ELISA, Real time-PCR and Western blot data were analyzed by ANOVA and Student $t$-test, with $\mathrm{p}<0.05$ as the minimum level of significance.

\section{Results}

\section{Increased survival of VIP KO vs WT mice after LPS administration}

We previously reported that female VIP KO mice exhibited a paradoxical resistance to EAE induction [27]. To determine if the resistance in these mice could potentially be explained by a defect in innate immunity, we injected LPS $(40 \mathrm{mg} / \mathrm{kg})$ i.p. to female WT and VIP KO mice and monitored their survival over time. The administration of this endotoxin triggers an acute inflammatory response leading to multiple organ dysfunction and subsequently death in wild type mice within two to three days. Figure 1A depicts the Kaplan-Meier analysis of WT vs. VIP KO survival of four experiments. In the first 24 hours after LPS injection, WT and VIP KO mice succumbed to LPS at a similar rate, with $50.0 \%$ and $58.6 \%$ survival for WT and VIP KO mice, respectively, and no significant differences between the two curves. After this time point, the mortality rates dramatically diverged, with few deaths occurring in the VIP KO group in the next 24 hours, and none after 48 hours post-injection. At the end of the study ( 5 days after LPS administration), a significantly higher overall survival of VIP KO mice $(44.8 \%)$ vs WT mice $(3.6 \%)$ was observed $(* * p=0.0049$; curve comparison Logrank test). For histopathological studies we chose the lung, a main target tissue for immune cell infiltration and inflammation in this model of LPS-induced endotoxemia. After LPS administration, lung inflammatory injury is characterized by patchy areas of neutrophilic infiltrates with thickening of the alveolar septae. Interestingly, we found a mild degree of basal inflammation in female VIP KO mice lungs $(0.5 \pm 0.29$ histological score) compared to those of WT controls (Figure 1B), as it has been previously reported in males [24]. However, in agreement with our clinical data, whereas the lungs of WT mice exhibited severe infiltration by immune cells 24 hours after LPS, those of the VIP KO mice were significantly less inflamed and exhibited a better preserved architecture (Figure 1B). Indeed, the average histopathological score post-LPS administration was $2.25 \pm 0.24$ for WT mice, vs $1.53 \pm 0.26$ for VIP KO mice $(* \mathrm{p}<0.05)$ (Figure 1C). Interestingly, we did not observe such a resistant clinical phenotype to LPS in VIP KO male mice (data not shown), suggesting that the effects of chronic absence of VIP could be sex dependent. In this sense, sex differences in the composition of the peritoneal cell population have been reported [29].

\section{Proinflammatory cytokine levels are reduced in sera and peritoneal suspensions of VIP KO mice after LPS administration}

LPS triggers the secretion of proinflammatory cytokines by a wide array of cell types as a part of the innate immune response. In order to determine if the ability of VIP KO mice to develop such a response was impaired, we measured the protein levels of TNF $\alpha$, IL-6 and IL12p40 in the sera and the peritoneal suspensions of LPS injected mice by ELISA. As shown in Figure 2, the levels of all these cytokines increased in the serum and peritoneal fluid of the WT mice in response to LPS as expected, with different timecourses. For $\mathrm{TNF} \alpha$, there was an early induction with a peak only 3 hours after the injection of LPS in both types of samples (3-fold increase compared to basal levels). For IL-6 and IL-12, the levels increased progressively reaching the maximum 6 hours after the induction of the disease (3-fold or higher fold increase over controls). Supporting the clinical phenotype of VIP KO mice, even though we found an increase of proinflammatory cytokines after LPS injection in the sera and peritoneal suspensions of these mice, the levels of these cytokines were lower than those in the WT mice. The reduction in cytokine levels was particularly striking for TNF $\alpha$ 3 hours post-injection $(0.42 \pm 0.05 \mathrm{ng} / \mathrm{ml}$ in WT vs $0.23 \pm 0.05 \mathrm{ng} / \mathrm{ml}$ in VIP $\mathrm{KO}$ mice; ${ }^{*} \mathrm{p}<0.05$ in peritoneal suspensions and $0.98 \pm 0.09 \mathrm{ng} / \mathrm{ml}$ in WT vs $0.41 \pm 0.08 \mathrm{ng} / \mathrm{ml}$ in VIP KO mice; ***p $<0.001$ in sera). In addition, we measured the levels of the antiinflammatory cytokine IL-10. As for the proinflammatory cytokines analyzed, the levels of IL-10 were lower in VIP KO mice than in WT mice at early time points, especially 3 hours after LPS administration. Because IL-10 plays a role in the resolution phase of inflammation, we also measured its levels at a later time point, 24 hours post-LPS injection, which is the time point after which the death rate decreased in the VIP KO group. Interestingly, the levels of IL-10 in both peritoneal 


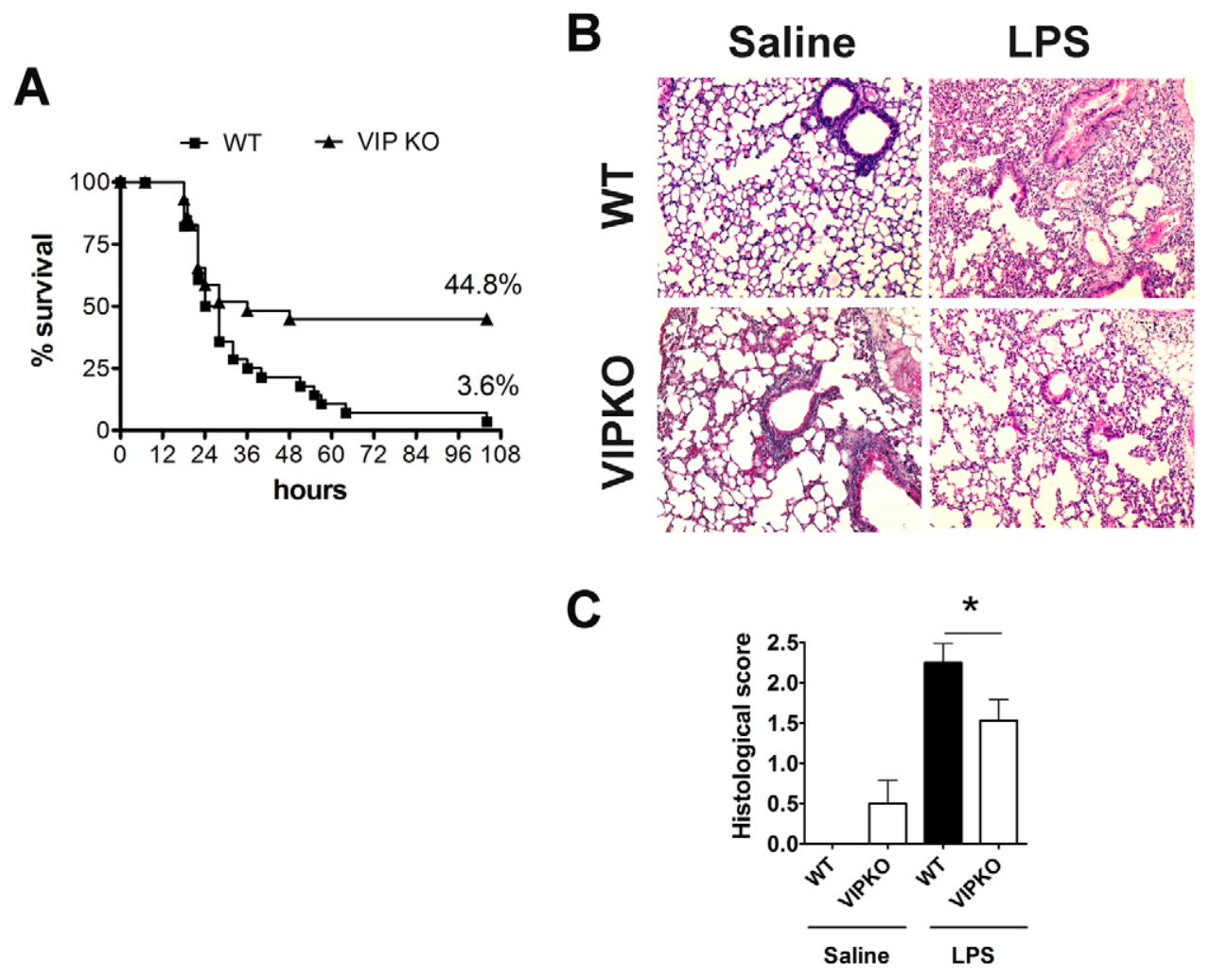

Figure 1. VIP KO mice exhibit reduced mortality and lung histopathology in response to LPS injection. Female WT (C57BL6) and VIP KO mice were injected i.p. with LPS (40 mg/Kg). A, Kaplan Meier curve analysis of survival cumulative of four experiments (total WT $n=29$; VIP KO $n=28$ ) (Curve comparison Logrank test ${ }^{* *} \mathrm{p}<0.01$ ). B, Representative sections of lungs from control (noninjected) or LPS-injected WT and VIP KO mice (24 hours post injection) stained with H\&E. C, Histological scores of LPS-injected WT vs. VIP KO mice (mean of two experiments; total WT $n=7$; VIP KO $\mathrm{n}=9$ ), 24 hours after LPS injection, scored from 0 to 3 according to the level of lung inflammation as described in Materials and Methods. (Student's $t-$ test $\left.{ }^{*} \mathrm{p}<0.05\right)$.

doi:10.1371/journal.pone.0036922.g001

suspensions and sera were significantly higher in VIP KO mice at this time $(* * * \mathrm{p}<0.001)$. The findings that the KO mice produced lower amounts of TNF $\alpha$, IL-6, IL-12p40 and IL-10 at early time points and produced more IL-10 at later time points suggest a depressed response of the innate arm of immunity, and correlate with the decreased mortality in the VIP KO mice.

\section{VIP KO mice express lower levels of proinflammatory mediators in peritoneal cells, spleen and lung}

Peritoneal cells are the first immune cell population exposed to LPS in this model. However, several studies have shown that LPS rapidly access the circulation after intraperitoneal administration and that its levels do not drop as quickly despite the clearing activity of liver cells [30]. Therefore, circulating LPS triggers a global inflammatory response whereby cells in different organs such as the spleen and lung become activated and produce proinflammatory mediators. These in turn promote immune cell migration and tissue infiltration. Immigrant immune cells can in turn amplify inflammation by producing more proinflammatory cytokines. As shown above, the systemic levels of proinflammatory cytokines were reduced in the VIP KO mice. In order to investigate the source of this deficiency, we measured by real time RT-PCR the mRNA levels of IL- 6 and TNF $\alpha$ as representative molecules of the inflammatory cascade, in peritoneal cells, spleen and lung two hours after LPS injection. As expected (Figure 3AC), the mRNA levels of these two proinflammatory markers were increased in all the WT mice tissues at 2 hours post-LPS injection.
However, the induction of these cytokines in VIP KO mice was much less pronounced than in WT mice in all organs analyzed. Only gene expression of IL-6 in the lung after LPS was not different between WT and VIP KO mice. Interestingly, the mRNA levels of TNF $\alpha$ in non-injected VIP KO lungs were higher than in the WT mice, in agreement with the mild basal inflammation found at the histological level in this organ in the KO mice $(* * p<0.01)$. Our results suggest that in the chronic absence of VIP, the LPS-induction of proinflammatory mediators was impaired.

\section{Peritoneal cells from VIP KO mice exhibit a reduced cytokine response to LPS in vitro}

In order to test whether the attenuated response to LPS in the VIP KO mice was due to an intrinsic defect in the ability of immune cells to mount an inflammatory response, we isolated and cultured whole peritoneal cells from WT and VIP KO mice in the presence or absence of LPS. Supernatants were collected to measure the levels of TNF $\alpha$ and IL- 6 by ELISA (Figure 4A, B). We found that 2 hours after LPS stimulation, WT cells secreted $\mathrm{TNF} \alpha$ and IL-6 as expected (Figure 4A). In comparison, VIP KO cells released lower cytokine levels ( ${ }^{*} \mathrm{p}<0.05$ for both cytokines). To assess the possibility that cytokine release may be delayed in VIP KO cells, we also collected the supernatants of similar cultures 16 hours after LPS stimulation (Figure 4B). Again, VIP KO cells produced significantly lower amounts of TNF $\alpha$ and IL-6 at this 


\section{Peritoneal suspension}

A
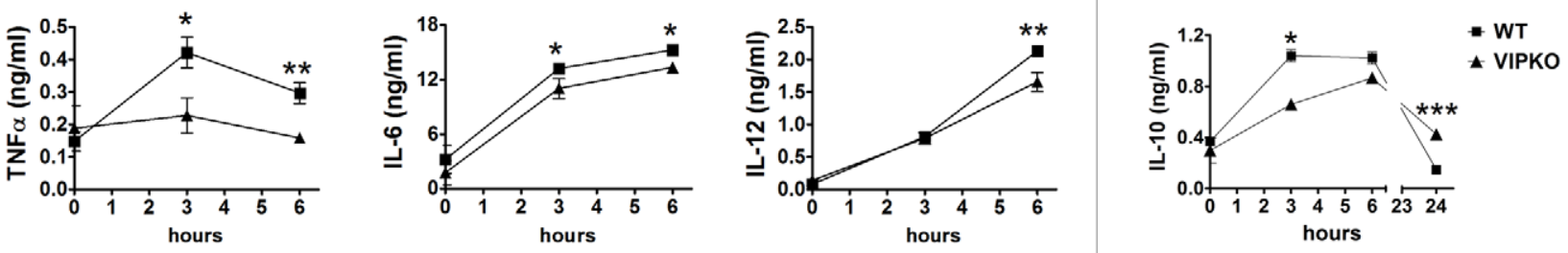

B
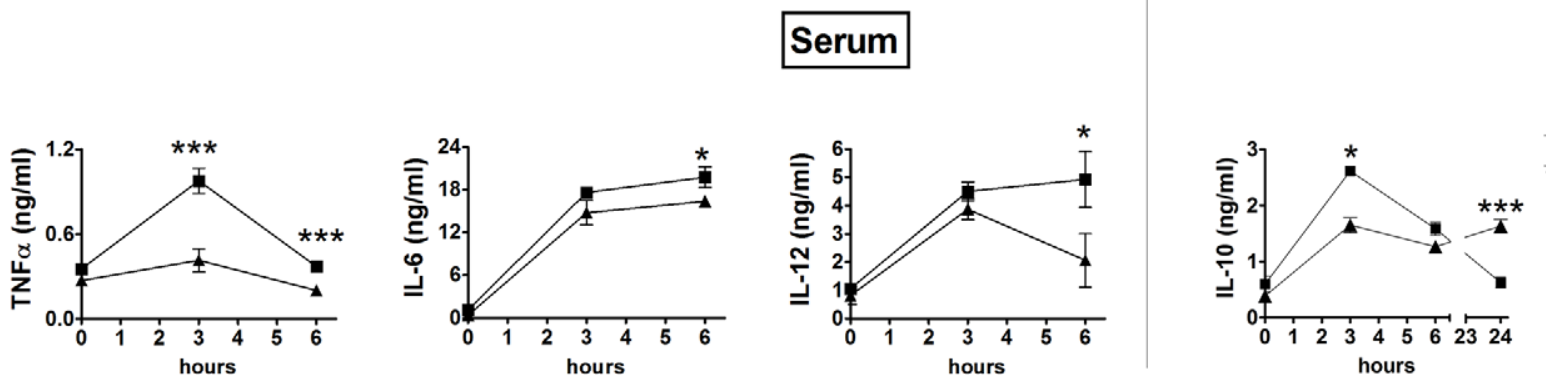

- WT

$\triangle$ VIPKO

Figure 2. VIP KO mice exhibit reduced levels of proinflammatory cytokines in the peritoneal fluid and serum. Female WT (C57BL6) $(n=6)$ and VIP KO mice $(n=6)$ were injected i.p. with LPS $(40 \mathrm{mg} / \mathrm{Kg})$, and serum and peritoneal suspensions were collected 0,3 and 6 (and also 24 for IL-10) hours post-injection. The levels of TNF $\alpha, \mathrm{IL}-6, \mathrm{IL}-12 \mathrm{p} 40$ and IL-10 were assessed by sandwich ELISA as described in Material and Methods. Student's $t$-test ${ }^{*} p<0.05 ;{ }^{* *} p<0.01$. One of three representative experiments is shown.

doi:10.1371/journal.pone.0036922.g002

time point $\left({ }^{*} \mathrm{p}<0.05\right)$. These in vitro experiments suggest that VIP KO mice may present a defect in immune cells to respond to LPS.

\section{NF- $\mathrm{KB}$ signaling is diminished in VIP KO peritoneal cells in vivo and in vitro after LPS administration}

The inflammatory cascade usually proceeds with the activation of the NF- $\kappa$ B pathway, which leads to the synthesis of chemokines and cytokines which amplify the immune response. In order to identify potential defects leading to the resistance of VIP KO mice to LPS, we studied the activity of this pathway in vitro and in vivo by Western blot (Figure 5A, B). As a readout of NF- $\kappa$ B activation, we studied the amount of phosphorylated-I $\mathrm{B}(\mathrm{p}-\mathrm{I} \kappa \mathrm{B})$ in protein extracts from whole peritoneal cells isolated from WT and VIP $\mathrm{KO}$ mice in basal conditions and 1 hour after LPS injection (Figure 5A). Interestingly, the levels of p-IкB were already lower in non-injected VIP KO mice compared to WT controls. The levels of $\mathrm{p}$-I $\mathrm{B}$ increased in both WT and VIP KO mice post-LPS injection. However, the levels of this signaling mediator after LPS were significantly lower in VIP-deficient mice, suggesting a reduced responsiveness of the NF- $\kappa \mathrm{B}$ pathway in these mice. In addition, we measured $\mathrm{p}-\mathrm{I} \kappa \mathrm{B}$ in peritoneal cells cultured with and without LPS as we did above for cytokine measurements (Figure 5B). Similar to the results found in vivo, whereas LPS treatment significantly elevated the levels of $\mathrm{p}-\mathrm{I} \kappa \mathrm{B}$ in peritoneal cells from WT mice $\left({ }^{*} \mathrm{p}<0.05\right)$, it only triggered a modest increase of $\mathrm{p}-\mathrm{I} \kappa \mathrm{B}$ in cells from VIP KO mice $(\mathrm{p}=0.15)$. Indeed, the levels of $\mathrm{p}-\mathrm{I} \kappa \mathrm{B}$ in cells treated with LPS where lower in VIP KO mice than in WT mice, although the difference was not significant $(p=0.08)$. The reduction of $\mathrm{NF}-\kappa \mathrm{B}$ signaling correlates with the impaired secretion of proinflammatory cytokines in the VIP KO mice.

\section{Discussion}

The antiinflammatory actions of VIP have been repeatedly demonstrated both in vitro and in vivo, suggesting the therapeutic potential of this peptide [11]. However, using the $\mathrm{MOG}_{35-55}$ immunization model, we recently reported that female VIP KO mice exhibited an unexpected resistance to EAE induction [27], suggesting that the activities of endogenous VIP in the immune system may be complex. During the pathogenesis of EAE, both the innate and adaptive arms of the immune system are involved [31]. Interestingly, we did not find a defect in the lymphocyte priming phase in the VIP KO mice in our prior EAE study, because T cells from immunized VIP KO mice showed robust responses to MOG in vitro on antigen rechallenge experiments [27]. In addition, it has been recently shown that VIP KO mice exhibited less weight loss and improved survival to murine CMV infection, a phenotype which was associated with enhanced adaptive antiviral cellular immunity [32]. Thus, the activation of CD4 and CD8 T cells following immunization does not appear to be impaired in these mice. On the other hand, adoptive transfer of $\mathrm{T}$ cells from $\mathrm{MOG}_{35-55}$-immunized WT mice induced EAE in WT but not VIP KO mice [27], suggesting that impairments in one or more cell types other than $\mathrm{T}$ cells might explain the EAE resistance in the latter mice. Here, we used the LPS model of endotoxemia and we found that VIP KO mice exhibited reduced mortality, less tissue injury, and impaired proinflammatory responses. Because the innate immune system is the major component in the pathogenesis of this model, these results suggest that these mice may exhibit a defect in this arm of immunity. We investigated potential deficiencies in TNF $\alpha$ and IL- 6 in these mice, as these are two cytokines critically involved in the acute inflammatory cascade. In this sense, $\mathrm{TNF} \alpha$ is rapidly produced by immune cells and is a major activator of the NF- $\mathrm{KB}$ pathway, which triggers the 

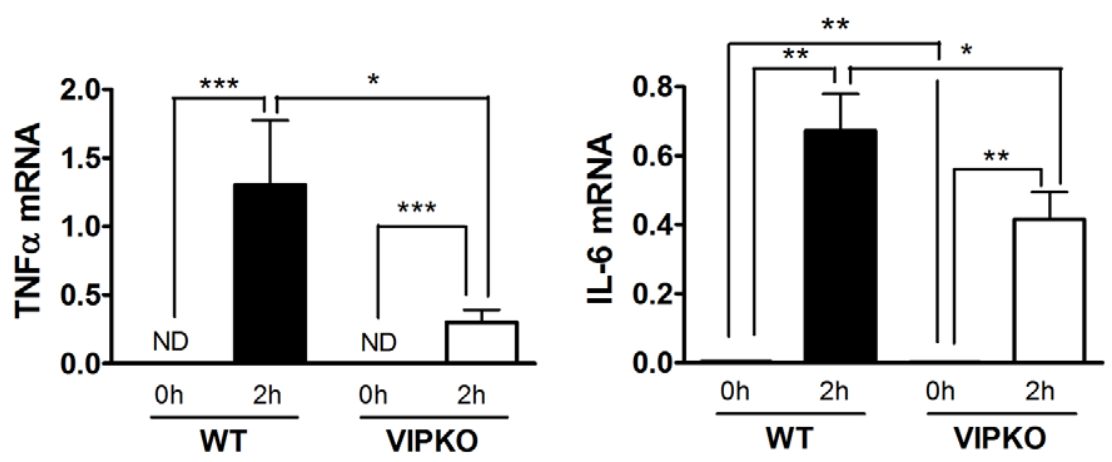

B

\section{Spleen}
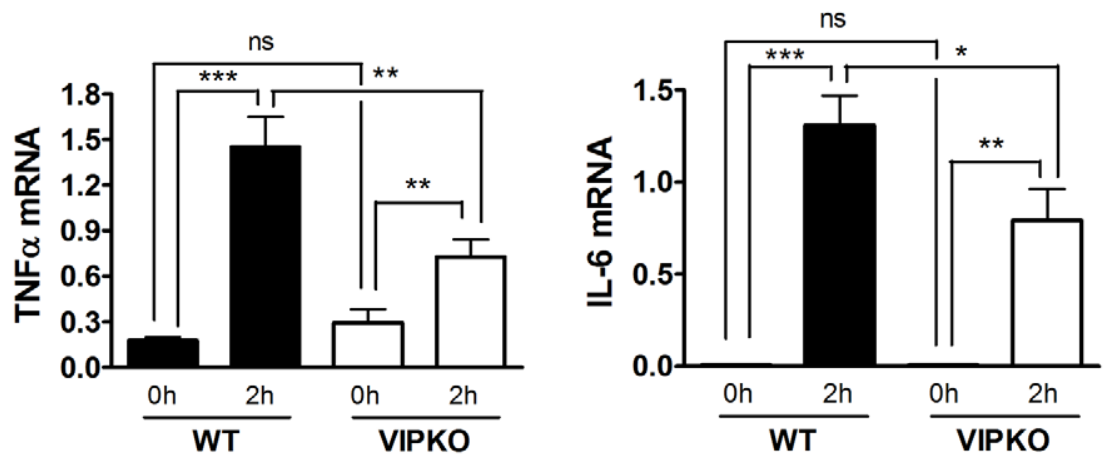

C

Lung
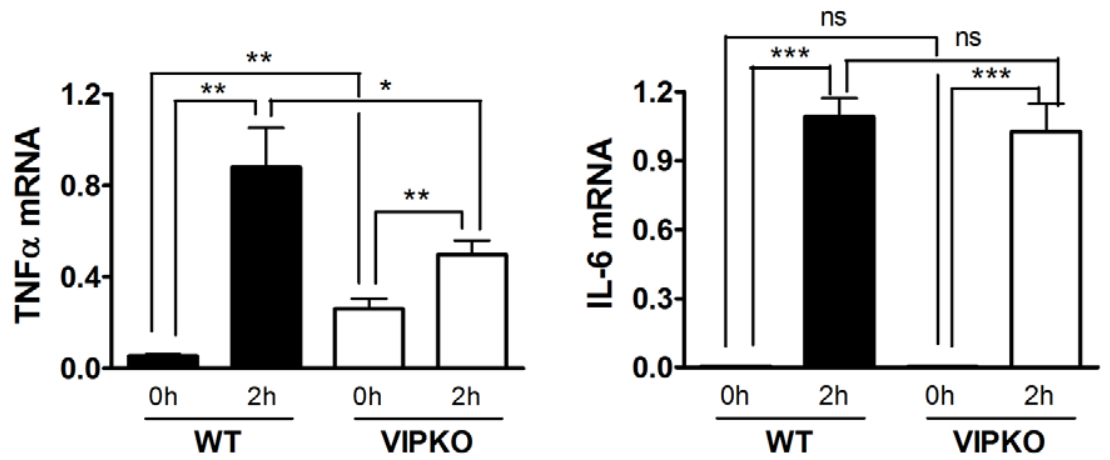

Figure 3. Levels of TNF $\alpha$ and IL- 6 gene expression in peritoneal cells, spleen and lung after LPS injection is reduced in VIP KO vs. WT mice. Female WT $(C 57 B L 6)(n=6)$ and VIP KO mice $(n=6)$ were injected i.p. with LPS $(40 \mathrm{mg} / \mathrm{Kg})$, and cells or tissues were collected 0 and 2 hours post-injection and stored at $-80^{\circ} \mathrm{C}$ until further analysis. RNA was extracted with the Trizol method, and the mRNA expressions of TNF $\alpha$ and IL- 6 were assessed by Real time RT-PCR. The mean values \pm SEM are shown $(n=6 / g r o u p)$. Student's $t$-test ${ }^{*} p<0.05$. doi:10.1371/journal.pone.0036922.g003

expression of chemokines and other proinflammatory cytokines, amplifying the immune response [33], IL-6, in addition to generally being an inflammatory cytokine, activates the coagulatory cascade, and if present in excessively high levels, can lead to disseminated intravascular coagulation, impaired reperfusion and death [34]. In our study, we found a reduction in the systemic levels of both TNF $\alpha$ and IL-6 in VIP KO mice injected with LPS. Although multiple cell types can produce these cytokines, our analysis of peritoneal cells infers that VIP KO mice may exhibit a defect in immune cells, most likely within the myeloid component, because the pool of peritoneal cells is highly represented by myeloid cells including monocytes, macrophages and granulocytic populations, and these cells are the main contributors to the rapid increase in TNF $\alpha$ and IL-6 levels in response to LPS. It has been shown that peritoneal cells play an important role in LPS-induced endotoxemia. First of all, this is the initial immune cell population exposed to the intraperitoneal LPS injection, and all cell components express TLRs. Moreover, it has been shown that peritoneal lavage performed before LPS administration to remove these cells reduces the elevation of serum TNF $\alpha$ and IL- 6 and mortality in mice [35]. Here, we found decreased TNF $\alpha$ and IL-6 mRNA levels in peritoneal cells from VIP KO mice injected with 
A
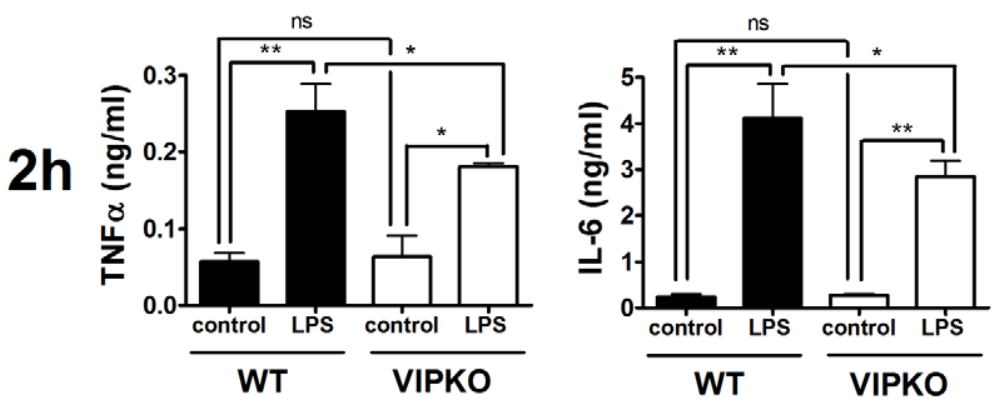

D
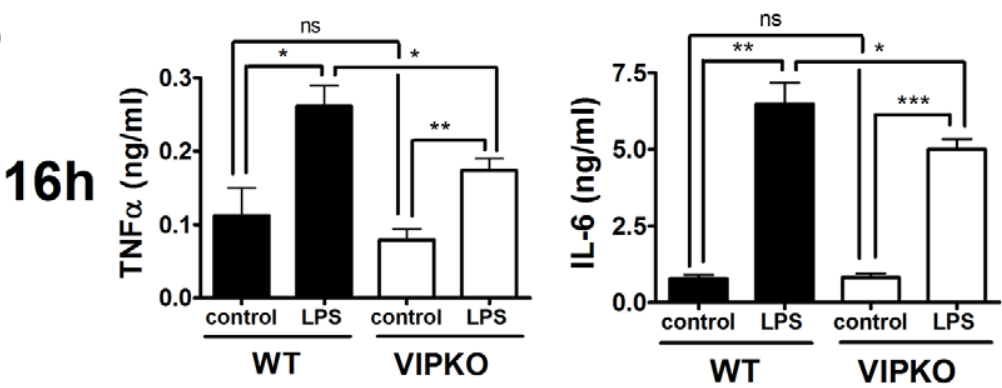

Figure 4. Peritoneal cells from VIP KO mice exhibit an intrinsic defect in cytokine response to LPS- administration. Peritoneal cells were collected from WT $(n=3)$ and VIP KO mice $(n=3)$, and cultured in complete RPMI in triplicate in the presence or absence of LPS $(10 \mathrm{ng} / \mathrm{ml})$. Supernatants were collected 2 (A) and $16 \mathrm{~h}$ (B) later, and stored at $-20^{\circ} \mathrm{C}$ for analysis of TNF $\alpha$ and IL- 6 levels by ELISA. Student's $t$-test ${ }^{*} p<0.05$; ${ }^{* *} \mathrm{p}<0.01 ; * * * \mathrm{p}<0.001$. Representative data are shown of four independent experiments. doi:10.1371/journal.pone.0036922.g004

A

IN VIVO

$\frac{\text { WT }}{\text { Oh } \quad 1 \mathrm{~h}} \stackrel{\text { VIP KO }}{\text { Oh } \quad 1 \mathrm{~h}}$

$-\frac{\text { P-IKB }}{\beta \text {-actin }}$

C

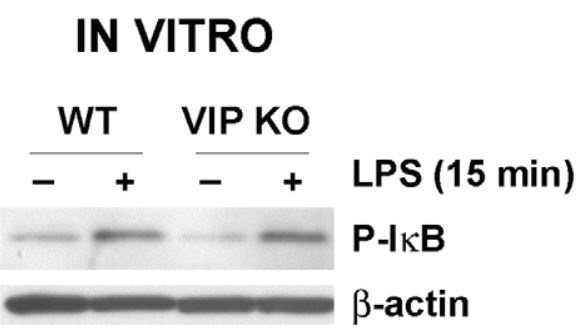

B

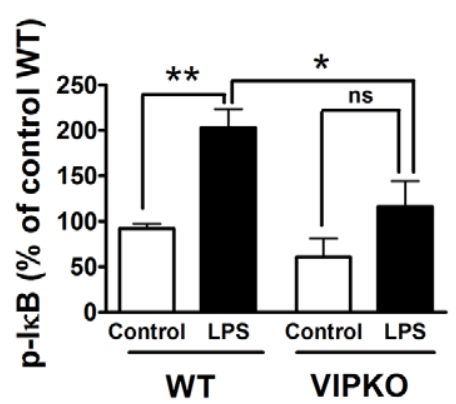

D

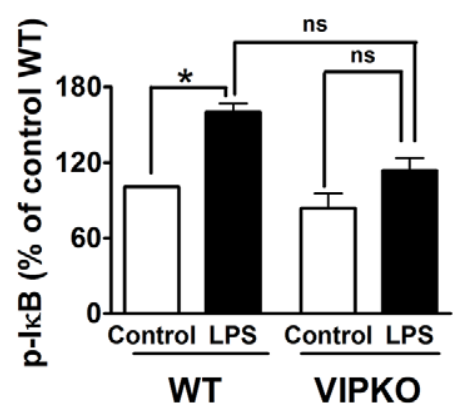

Figure 5. LPS-induced activation of the NF-KB pathway is diminished in VIP KO mice. Activation of the NF- $\kappa B$ signaling pathway was at several time point determined by measurement of $p-I \kappa B$ protein levels by Western blot in (A, B) peritoneal cells isolated from WT and VIP KO mice without or 1 hour after injection of LPS $(40 \mathrm{mg} / \mathrm{Kg})$, and $(C, D)$ peritoneal cells isolated from WT and VIP KO mice and stimulated in culture with or without LPS $(10 \mathrm{ng} / \mathrm{ml})$. Bar graphs represent the quantification of band density. Student's $t$-test ${ }^{*} \mathrm{p}<0.05, \mathrm{~ns}=$ not significant. A representative blot of three experiments is shown.

doi:10.1371/journal.pone.0036922.g005 
LPS, and that these cells responded poorly to LPS in vitro. This reduction suggests that the intrinsic ability of myeloid cells to elicit an inflammatory response when exposed to LPS may be impaired in the chronic absence of VIP.

Could a myeloid deficit in VIP KO mice contribute to their EAE resistance? The proinflammatory activities of autoreactive lymphocytes with Th1 and Th17 profiles have been shown to be essential for EAE development [36]. However, myeloid cells have been proposed as the ultimate effector cells that lead to the CNS damage [31]. Myeloid cells may contribute to the pathogenesis of EAE by secreting proinflammatory cytokines and chemokines, but they may also mediate tissue damage in this model by releasing enzymes that contribute to myelin destruction. For example, it has been shown that metalloproteases, such as MMP-2, released by macrophages cells in the CNS during EAE, enable the breakdown of the blood brain barrier and the infiltration of the CNS parenchyma by immune cells [37]. In addition to the resident microglia, abundant numbers of macrophages infiltrate the CNS during EAE. Depletion of macrophages with liposomal dichloromethylene diphosphonate $\left(\mathrm{Cl}_{2} \mathrm{MDP}\right)$ after EAE induction but before the appearance of clinical symptoms, led to the suppression of full EAE development [38]. Because this treatment eliminates bone marrow-derived macrophages leaving other populations such as microglia intact [39], the results highlight a crucial role for newly recruited macrophages for the development of the disease. Supporting the relevance of the proinflammatory role of macrophages in EAE, mice deficient in the macrophage chemokine CGL-1 (MCP-1) [40] and its receptor CGR-2 [41] are resistant to $\mathrm{EAE}$, and genetic activation of the $\mathrm{NF}-\kappa \mathrm{B}$ pathway specifically in myeloid cells led to a more severe course of the disease [42]. We have found an impairment of NF- $\kappa \mathrm{B}$ pathway signaling in VIP KO mice peritoneal cells, indicating an intrinsic defect in the response of these cells to at least some types of inflammatory stimuli. Several innate immune receptors activate the NF- $\kappa \mathrm{B}$ pathway, including members of the IL-1R/TLR and TNF-R 1 superfamilies. In addition, LPS directly activates NF- $\kappa \mathrm{B}$ signaling through TLR4, which in turn triggers the expression of proinflammatory cytokines such as TNF $\alpha$, IL-6 and IL- 12 . Although the specific mechanisms by which the NF- $\kappa \mathrm{B}$ activation is impaired in VIP KO mice remain to be elucidated, this could be potentially due to abnormalities in TLR4 signaling in mice due to chronic absence of VIP.

At this point, we can only hypothesize why the lack of VIP results in decreased inflammation in the models of EAE and LPSinduced endotoxemia. At first glance, the data in this report contradicts the multiple existing studies demonstrating that VIP inhibits the release of proinflammatory mediators by murine

\section{References}

1. Medzhitov R (2008) Origin and physiological roles of inflammation. Nature 454: 428-435.

2. Jean-Baptiste E (2007) Cellular mechanisms in sepsis. J Intensive Care Med 22: $63-72$.

3. Silva MT (2010) When two is better than one: macrophages and neutrophils work in concert in innate immunity as complementary and cooperative partners of a myeloid phagocyte system. J Leukoc Biol 87: 93-106.

4. Sadik CD, Kim ND, Luster AD (2011) Neutrophils cascading their way to inflammation. Trends Immunol 32: 452-460.

5. Stearns-Kurosawa DJ, Osuchowski MF, Valentine C, Kurosawa S, Remick DG (1991) The pathogenesis of sepsis. Annu Rev Pathol 6: 19-48.

6. Kawai T, Akira S (2010) The role of pattern-recognition receptors in innate immunity: update on Toll-like receptors. Nat Immunol 11: 373-384.

7. Lu YC, Yeh WC, Ohashi PS (2008) LPS/TLR4 signal transduction pathway. Cytokine 42: 145-151.

8. Kawai T, Akira S (2007) Signaling to NF-kappaB by Toll-like receptors. Trends Mol Med 13: 460-469.

9. Serhan CN, Savill J (2005) Resolution of inflammation: the beginning programs the end. Nat Immunol 6: 1191-1197. macrophages $[18,21,43,44]$. In these studies, VIP was added simultaneously or after the inflammatory stimulus. However, a few studies have shown that VIP can stimulate various aspects of the immune response under certain circumstances, for example when added to resting cells in culture. In this regard, VIP promoted the secretion of IL-6 by resting peritoneal macrophages in vitro, whereas it inhibited the production of this cytokine in the presence of LPS, suggesting that this peptide may exhibit dual actions depending on the activation status of the cell $[45,46]$. In other organs, such as the anterior pituitary and bone marrow, VIP also stimulates IL-6 production, which in turn stimulates the release of pituitary hormones and the growth and differentiation of hematopoietic cells in the bone marrow [47]. Interestingly, Yadav et al have recently shown that VPAC1 KO mice present milder DSS-induced colitis compared to WT mice, suggesting that VIP may exert proinflammatory actions through this receptor [30]. Although these results suggest an impaired functionality of VIP KO of VPAC1 KO immune cells, other possibilities such as compensation by other factors such as peptides/hormones or their receptors may occur in mice when these molecules are chronically absent, and cannot be ruled out at this point. For example, we found a late increase in the systemic levels of IL-10 which could potentially contribute to the increased survival in the $\mathrm{KO}$ mice, although the importance of this mild induction needs to be further studied. Finally, an interesting finding was that despite the reduced mortality in VIP KO mice injected with LPS, we found a mild lung histological inflammation and higher levels of TNF $\alpha$ mRNA in this tissue in VIP KO mice in basal conditions. Szema et al previously reported similar results in VIP KO males, and agrees with the purported antiinflammatory actions of VIP [24]. It has been shown that prolonged administration of a low dose of LPS causes mild-degree inflammation which results in later poor responsiveness to LPS, a phenomenon known as "endotoxin tolerance" [48]. In would be interesting to determine if a continuous basal inflammation in VIP KO mice may be related to their decreased response to LPS. In conclusion, the unexpected decreased response of female VIP KO mice to LPS highlights the complexity of the actions of this peptide. This will need to be taken into account when therapies are designed to target this signaling pathway in inflammatory diseases.

\section{Author Contributions}

Conceived and designed the experiments: CA YVT GCL HN JAW. Performed the experiments: CA YVT GCL HN. Analyzed the data: CA JAW. Contributed reagents/materials/analysis tools: CA YVT GCL HN JAW. Wrote the paper: CA YVT JAW.

10. Delgado M, Ganea D (2008) Anti-inflammatory neuropeptides: a new class of endogenous immunoregulatory agents. Brain Behav Immun 22: 1146-1151.

11. Delgado M, Pozo D, Ganea D (2004) The significance of vasoactive intestinal peptide in immunomodulation. Pharmacol Rev 56: 249-290.

12. Gomariz RP, Martinez C, Abad C, Leceta J, Delgado M (2001) Immunology of VIP: a review and therapeutical perspectives. Curr Pharm Des 7: 89-111.

13. Laburthe M, Couvineau A, Tan V (2007) Class II G protein-coupled receptors for VIP and PACAP: structure, models of activation and pharmacology. Peptides 28: 1631-1639.

14. Delgado M, Ganea D (2001) Inhibition of endotoxin-induced macrophage chemokine production by vasoactive intestinal peptide and pituitary adenylate cyclase-activating polypeptide in vitro and in vivo. J Immunol 167: 966-975.

15. Ganea D, Delgado M (2001) Neuropeptides as modulators of macrophage functions. Regulation of cytokine production and antigen presentation by VIP and PACAP. Arch Immunol Ther Exp (Warsz) 49: 101-110.

16. Delgado M, Abad C, Martinez C, Leceta J, Gomariz RP (2001) Vasoactive intestinal peptide prevents experimental arthritis by downregulating both autoimmune and inflammatory components of the disease. Nat Med 7: $563-568$. 
17. Abad C, Martinez C, Juarranz MG, Arranz A, Leceta J, et al. (2003) Therapeutic effects of vasoactive intestinal peptide in the trinitrobenzene sulfonic acid mice model of Crohn's disease. Gastroenterology 124: 961-971.

18. Delgado M, Martinez C, Pozo D, Calvo JR, Leceta J, et al. (1999) Vasoactive intestinal peptide (VIP) and pituitary adenylate cyclase-activation polypeptide (PACAP) protect mice from lethal endotoxemia through the inhibition of TNFalpha and IL-6. J Immunol 162: 1200-1205.

19. Gonzalez-Rey E, Fernandez-Martin A, Chorny A, Martin J, Pozo D, et al. (2006) Therapeutic effect of vasoactive intestinal peptide on experimental autoimmune encephalomyelitis: down-regulation of inflammatory and autoimmune responses. Am J Pathol 168: 1179-1188.

20. Brandtzaeg P, Oktedalen O, Kierulf P, Opstad PK (1989) Elevated VIP and endotoxin plasma levels in human gram-negative septic shock. Regul Pept 24: 37-44.

21. Delgado M, Pozo D, Martinez C, Leceta J, Calvo JR, et al. (1999) Vasoactive intestinal peptide and pituitary adenylate cyclase-activating polypeptide inhibit endotoxin-induced TNF-alpha production by macrophages: in vitro and in vivo studies. J Immunol 162: 2358-2367.

22. Ait-Ali D, Stroth N, Sen JM, Eiden LE. PACAP-cytokine interactions govern adrenal neuropeptide biosynthesis after systemic administration of LPS. Neuropharmacology 58: 208-214.

23. Martinez C, Delgado M, Abad C, Gomariz RP, Ganea D, et al. (1999) Regulation of VIP production and secretion by murine lymphocytes. J Neuroimmunol 93: 126-138.

24. Szema AM, Hamidi SA, Lyubsky S, Dickman KG, Mathew S, et al. (2006) Mice lacking the VIP gene show airway hyperresponsiveness and airway inflammation, partially reversible by VIP. Am J Physiol Lung Cell Mol Physiol 291: L880-886.

25. Colwell CS, Michel S, Itri J, Rodriguez W, Tam J, et al. (2003) Disrupted circadian rhythms in VIP- and PHI-deficient mice. Am J Physiol Regul Integr Comp Physiol 285: R939-949.

26. Hamidi SA, Prabhakar S, Said SI (2008) Enhancement of pulmonary vascular remodelling and inflammatory genes with VIP gene deletion. Eur Respir J 31: 135-139.

27. Abad C, Tan YV, Lopez R, Nobuta H, Dong H, et al. (2010) Vasoactive intestinal peptide loss leads to impaired CNS parenchymal T-cell infiltration and resistance to experimental autoimmune encephalomyelitis. Proc Natl Acad Sci U S A 107: 19555-19560.

28. Matute-Bello G, Winn RK, Jonas M, Chi EY, Martin TR, et al. (2001) Fas (CD95) induces alveolar epithelial cell apoptosis in vivo: implications for acute pulmonary inflammation. Am J Pathol 158: 153-161.

29. Scotland RS, Stables MJ, Madalli S, Watson P, Gilroy DW (2011) Sex differences in resident immune cell phenotype underlie more efficient acute inflammatory responses in female mice. Blood 118: 5918-5927.

30. Hansen MK, Nguyen KT, Fleshner M, Goehler LE, Gaykema RP, et al. (2000) Effects of vagotomy on serum endotoxin, cytokines, and corticosterone after intraperitoneal lipopolysaccharide. Am J Physiol Regul Integr Comp Physiol 278: R331-336.

31. Batoulis H, Addicks K, Kuerten S (2010) Emerging concepts in autoimmune encephalomyelitis beyond the CD4/T(H)1 paradigm. Ann Anat 192: 179-193.

32. Li JM, Southerland L, Hossain MS, Giver CR, Wang Y, et al. (2011) Absence of vasoactive intestinal peptide expression in hematopoietic cells enhances Th1 polarization and antiviral immunity in mice. J Immunol 187: 1057-1065.
33. Beutler B, Cerami A (1988) Tumor necrosis, cachexia, shock, and inflammation: a common mediator. Annu Rev Biochem 57: 505-518.

34. ten Cate H (2000) Pathophysiology of disseminated intravascular coagulation in sepsis. Crit Care Med 28: S9-11.

35. Zhao K, Kirman I, Tschepen I, Schwab R, Weksler ME (1997) Peritoneal lavage reduces lipopolysaccharide-induced elevation of serum TNF-alpha and IL-6 mortality in mice. Inflammation 21: 379-390.

36. El-behi M, Rostami A, Ciric B (2010) Current views on the roles of Th1 and Th17 cells in experimental autoimmune encephalomyelitis. J Neuroimmune Pharmacol 5: 189-197.

37. Agrawal S, Anderson P, Durbeej M, van Rooijen N, Ivars F, et al. (2006) Dystroglycan is selectively cleaved at the parenchymal basement membrane at sites of leukocyte extravasation in experimental autoimmune encephalomyelitis. J Exp Med 203: 1007-1019.

38. Huitinga I, van Rooijen N, de Groot CJ, Uitdehaag BM, Dijkstra CD (1990) Suppression of experimental allergic encephalomyelitis in Lewis rats after elimination of macrophages. J Exp Med 172: 1025-1033.

39. Bauer J, Huitinga I, Zhao W, Lassmann H, Hickey WF, et al. (1995) The role of macrophages, perivascular cells, and microglial cells in the pathogenesis of experimental autoimmune encephalomyelitis. Glia 15: 437-446.

40. Huang DR, Wang J, Kivisakk P, Rollins BJ, Ransohoff RM (2001) Absence of monocyte chemoattractant protein 1 in mice leads to decreased local macrophage recruitment and antigen-specific $\mathrm{T}$ helper cell type 1 immune response in experimental autoimmune encephalomyelitis. J Exp Med 193: 713-726.

41. Fife BT, Huffnagle GB, Kuziel WA, Karpus WJ (2000) CG chemokine receptor 2 is critical for induction of experimental autoimmune encephalomyelitis. J Exp Med 192: 899-905.

42. Ellrichmann G, Thone J, Lee DH, Rupec RA, Gold R, et al. (2012) Constitutive activity of NF-kappa B in myeloid cells drives pathogenicity of monocytes and macrophages during autoimmune neuroinflammation. J Neuroinflammation 9: 15.

43. Delgado M, Munoz-Elias EJ, Gomariz RP, Ganea D (1999) VIP and PACAP inhibit IL-12 production in LPS-stimulated macrophages. Subsequent effect on IFNgamma synthesis by T cells. J Neuroimmunol 96: 167-181.

44. Delgado M, Munoz-Elias EJ, Gomariz RP, Ganea D (1999) Vasoactive intestinal peptide and pituitary adenylate cyclase-activating polypeptide prevent inducible nitric oxide synthase transcription in macrophages by inhibiting NFkappa B and IFN regulatory factor 1 activation. J Immunol 162: 4685-4696.

45. Martinez C, Delgado M, Pozo D, Leceta J, Calvo JR, et al. (1998) Vasoactive intestinal peptide and pituitary adenylate cyclase-activating polypeptide modulate endotoxin-induced IL- 6 production by murine peritoneal macrophages. J Leukoc Biol 63: 591-601.

46. Martinez C, Delgado M, Pozo D, Leceta J, Calvo JR, et al. (1998) VIP and PACAP enhance IL-6 release and mRNA levels in resting peritoneal macrophages: in vitro and in vivo studies. J Neuroimmunol 85: 155-167.

47. Cai Y, Xin X, Shim GJ, Mokuno Y, Uehara H, et al. (1997) Pituitary adenylate cyclase activating polypeptide (PACAP) and vasoactive intestinal peptide (VIP) stimulate interleukin-6 production through the third subtype of PACAP/VIP receptor in rat bone marrow-derived stromal cells. Endocrinology 138: 2515-2520.

48. Biswas SK, Lopez-Collazo E (2009) Endotoxin tolerance: new mechanisms, molecules and clinical significance. Trends Immunol 30: 475-487. 